\title{
Antibody Drug Conjugates: Nonclinical Safety Considerations
}

\author{
Mary Jane Masson Hinrichs, ${ }^{1,2}$ and Rakesh Dixit ${ }^{1}$
}

Received 26 February 2015; accepted 14 May 2015; published online 30 May 2015

\begin{abstract}
Antibody drug conjugates (ADCs) are biopharmaceutical molecules consisting of a cytotoxic small molecule covalently linked to a targeted protein carrier via a stable cleavable or noncleavable linker. The process of conjugation yields a highly complex molecule with biochemical properties that are distinct from those of the unconjugated components. The impact of these biochemical differences on the safety and pharmacokinetic (PK) profile of the conjugate must be considered when determining the types of nonclinical safety studies required to support clinical development of ADCs. The hybrid nature of ADCs highlights the need for a science-based approach to safety assessment that incorporates relevant aspects of small and large molecule testing paradigms. This thinking is reflected in current regulatory guidelines, where sections pertaining to conjugates allow for a flexible approach to nonclinical safety testing. The aim of this article is to review regulatory expectations regarding early assessment of nonclinical safety considerations and discuss how recent advances in our understanding of ADCmediated toxicity can be used to guide the types of nonclinical safety studies needed to support ADC clinical development. The review will also explore nonclinical testing strategies that can be used to streamline ADC development by assessing the safety and efficacy of next generation ADC constructs using a rodent screen approach.
\end{abstract}

KEY WORDS: antibody drug conjugates; regulatory guidance; safety assessment; therapeutic index

\section{INTRODUCTION}

Antibody drug conjugates (ADCs) are emerging as a promising class of biopharmaceutical anti-cancer agents following the recent approvals of Kadcyla ${ }^{\circledR}$ and Adcetris ${ }^{\circledR}$ (1). From the nonclinical safety perspective, ADCs present unique challenges to standard toxicology testing due to the complex nature of the conjugate, which consists of both small and large molecule components. Specifically, the process of conjugating a highly potent cytotoxic small molecule (warhead) to a highly targeted monoclonal antibody $(\mathrm{mAb})$ yields a hybrid molecule with a toxicity profile that is distinct from that of the individual components. This necessitates a science-driven approach to safety assessment that considers the unique biochemical properties of the conjugate when determining the types of nonclinical studies needed to support first in human trials with ADCs. The need for an adaptive approach is reflected in current regulatory guidelines-International Conference on Harmonization (ICH)S6(R1) and

\footnotetext{
${ }^{1}$ Department of Translational Sciences, MedImmune LLC, One MedImmune Way, Gaithersburg, Maryland 20878, USA.

${ }^{2}$ To whom correspondence should be addressed. (e-mail: hinrichsm@medimmune.com)
}

ICHS9-where sections pertaining to conjugates allow for a flexible approach to nonclinical safety testing $(2,3)$. The primary aim of this article is to review regulatory expectations regarding early assessment of nonclinical safety considerations and discuss how recent advances in our understanding of ADC behavior and mechanisms of toxicity can be used to guide the types of nonclinical safety studies needed to support ADC clinical development. The topics discussed in this review reflect a science-based approach to the interpretation of existing relevant regulatory guidelines and available literature and is not meant to provide a blueprint for nonclinical safety assessment of ADCs. Additional details pertaining to the design and conduct of nonclinical programs to support clinical development of ADCs can be found in a recent comprehensive industry white paper on this topic (4).

In addition to the nonclinical safety evaluation of clinical candidate ADCs, there is also a growing need within the industry to evaluate the safety of rapidly evolving ADC technologies early in the discovery process. Recent engineering efforts to improve the therapeutic index (TI) of next generation ADCs have resulted in an explosion of new technologies with the potential to significantly impact safety and pharmacokinetic (PK). Thus as a secondary aim, this 
paper will discuss screening strategies that can be used to streamline safety evaluation of novel ADC technologies.

\section{UNIQUE CHARACTERISTICS OF ADCS THAT IMPA CT SAFETY}

ADCs combine three components to create a complex molecule consisting of a potent cytotoxic molecule chemically linked to a tumor-targeting monoclonal antibody via a cleavable or noncleavable linker. As described above, the conjugated form of the antibody has unique biochemical properties that significantly alter the safety profile of the conjugate compared to the individual components (Table I).

One of the unique characteristics of ADCs is the PK profile; conjugation significantly impacts half-life, clearance, elimination, and biodistribution of the unconjugated components (5). Elimination of unconjugated antibodies involves catabolic breakdown and/or target-mediated clearance pathways while small molecule warheads are typically cleared through hepatic and/or renal pathways (6). In contrast, elimination of an ADC involves properties of both large and small molecules. The conjugate must first be broken down by catabolism to yield ADC catabolites or undergo deconjugation to yield naked antibody and warhead components (5). Once broken down, the antibody is degraded into amino acids for recycling while the warhead undergoes renal and/or hepatic-mediated clearance. This difference in elimination also impacts half-life of the ADC. While the half-life of an unconjugated monoclonal antibody typically ranges from 1 to 3 weeks (7), the half-life of an ADC is generally only 2 to 5 days (8). This effect on half-life is thought to be related to the overall stability of the conjugate (9). This is supported by the observation that trastuzumab conjugated to DM1 via a cleavable linker was cleared approximately twofold faster than trastuzumab employing a more stable noncleavable linker (10). The impact of conjugation on the PK profile of the warhead is even greater; conjugation can extend the halflife of a warhead from hours to days $(5,11)$. Biodistribution of the unconjugated components is also significantly impacted by conjugation (5). Antibodies are largely restricted to plasma space with slow diffusion into tissues $(12,13)$ while small molecule warheads generally have a much higher volume of distribution depending on chemical properties of the molecule. The conjugation process limits warhead to antibody-restricted plasma space and target-expressing cells $(14,15)$. This change in biodistribution can change the toxicity profile of the warhead by altering distribution of the cytotoxic agent to tissues in which antibodies are known to accumulate-i.e., liver, lungs, kidney, and skin-and to normal tissues expressing the tumor target $(16,17)$.

Another key difference between ADCs and therapeutic mAbs is the antibody itself. While both ADCs and therapeutic mAbs display high selectivity for the target, antibodies used as part of an ADC are typically selected for their ability to undergo rapid internalization in order to elicit tumor cell-specific killing (18). Therapeutic mAbs, however, are selected based on inherent effector function, i.e., blocking/agonist and/or immune-modulating activity (19).

\section{MECHANISMS OF TOXICITY}

The concept of on and off-target toxicity-i.e., targetdependent and target-independent toxicity - is central to our understanding of ADC-mediated toxicity (Table II) and should be considered when deciding on the types of nonclinical studies that will provide the best dataset to evaluate ADC safety.

\section{On-Target Toxicity}

The ideal ADC target is highly expressed on tumor cells and minimally expressed or absent on normal cells. However, in reality, nearly all target proteins have some degree of normal tissue expression, which can be a major obstacle to ADC development (20). A well-known example of on-target toxicity is bivatuzumab mertansine, an anti-human CD44v6 antibody conjugated to DM1 (21). Immunohistochemistry (IHC) studies demonstrated CD44v6 staining in keratinocytes and epithelial cells in the cornea and tonsils (22). The initial phase 1 trial with bivatuzumab mertansine was halted following observations of dose-dependent skin toxicity, ending in a fatal case of toxic epidermal necrolysis (21). This finding-characterized by keratinocyte apoptosis and desquamation-was thought to be related to CD44v6 expression in the skin; other DM1 ADCs have not shown similar toxicities in the clinic (23). However, the potential for ontarget toxicity can be difficult to predict based on expression patterns alone (Table III). In the case of Kadcyla ${ }^{\circledR}$ (T-DM1), the major dose-limiting toxicities observed in clinical trials were thrombocytopenia and elevated liver enzymes despite significant target expression on cardiac myocytes and epithelial cells in GI tract, lung, skin, and breast (40-42). It is clear from this example that on-target toxicity is not driven solely by target expression and that other factors-e.g., proliferative/

Table I. Unique Characteristics of ADCs vs. Unconjugated Components

\begin{tabular}{|c|c|c|c|}
\hline & ADCs & Warhead & Antibodies \\
\hline Half-life & Intermediate half-life & Rapid half-life & Prolonged half-life \\
\hline Clearance & $\begin{array}{l}\text { Catabolism/target-mediated clearance of } \mathrm{mAb} \text {; } \\
\text { hepatic and/or renal elimination of warhead }\end{array}$ & $\begin{array}{l}\text { Hepatic and/or } \\
\text { renal elimination }\end{array}$ & $\begin{array}{l}\text { Catabolism/target-mediated } \\
\text { clearance }\end{array}$ \\
\hline Biodistribution & Plasma volume & Variable & Plasma volume \\
\hline Dosing schedule & Intermittent & Intermittent & Maintain steady state \\
\hline Target interaction & High affinity; rapid internalization & N/A & $\begin{array}{l}\text { High affinity; blocking or } \\
\text { agonist activity }\end{array}$ \\
\hline
\end{tabular}


Table II. Mechanisms of ADC Toxicity

\begin{tabular}{|c|c|c|}
\hline & Mechanism(s) & Safety assessment \\
\hline On-target & $\begin{array}{l}\text { Binding/internalization of ADC in target-expressing } \\
\text { normal cells }\end{array}$ & $\begin{array}{l}\text { In vivo toxicity must be evaluated in crossreactive species; } \\
\text { requires understanding of normal tissue target expression }\end{array}$ \\
\hline Off-target & $\begin{array}{l}\text { Instability of conjugate; nonspecific uptake into normal } \\
\text { cells (i.e., Fc } \gamma \text { receptors, FcRn binding, pinocytosis, } \\
\text { etc.); nonspecific binding of antibody to normal cells }\end{array}$ & $\begin{array}{l}\text { Off-target toxicity can be evaluated in non cross-reactive } \\
\text { species }\end{array}$ \\
\hline
\end{tabular}

Table III. Examples of ADC-Mediated On- and Off-Target Toxicity in the Clinic

\begin{tabular}{|c|c|c|c|c|}
\hline ADC & Target & Warhead & Normal tissue expression & Dose-limiting toxicities \\
\hline AGS-16M8F & ENPP3 & MMAF & $\begin{array}{l}\text { Gl tract (glandular cells), lung } \\
\text { (respiratory epithelial cells), kidney } \\
\text { tubules }\end{array}$ & Thrombocytopenia $(24,25)$ \\
\hline ASG-5ME & SLC44A4 & MMAE & $\begin{array}{l}\text { Apical expression in polarized cells } \\
\text { in kidney and Gl tract }\end{array}$ & $\begin{array}{l}\text { Neutropenia; GI toxicity; } \\
\text { neuropathy (26) }\end{array}$ \\
\hline AVE9633 & CD33 & DM4 & $\begin{array}{l}\text { BM hematopoietic cells } \\
\text { lymphocytes, GI tract (epithelial } \\
\text { and glandular) }\end{array}$ & Hepatotoxicity (27) \\
\hline BT-062 & CD138 & DM4 & $\begin{array}{l}\text { B cells, epithelial cells in GI tract, } \\
\text { liver, skin, kidney, pancreas, and } \\
\text { prostate }\end{array}$ & $\begin{array}{l}\text { Hand/foot syndrome; } \\
\text { mucositis }(28,29)\end{array}$ \\
\hline CDX-011 & GPNMB & MMAE & $\begin{array}{l}\text { Melanocytes, osteoblasts, retinal } \\
\text { epithelial cells }\end{array}$ & $\begin{array}{l}\text { Rash (hand/foot and TEN); } \\
\text { neutropenia; peripheral } \\
\text { neuropathy (30) }\end{array}$ \\
\hline CMC544 & $\mathrm{CD} 22$ & Calicheamicin & B cells & $\begin{array}{l}\text { Thrombocytopenia, } \\
\text { Iymphopenia, neutropenia ( } 31 \text { ) }\end{array}$ \\
\hline DCDT2980S & $\mathrm{CD} 22$ & MMAE & B cells, GI tract (glandular cells) & $\begin{array}{l}\text { Neutropenia; sensory } \\
\text { neuropathy (32) }\end{array}$ \\
\hline IMGN242 & $\begin{array}{l}\text { MUC1 } \\
\text { (CanAg) }\end{array}$ & DM4 & Mutated form of MUC1 on tumor & Ocular toxicity (keratitis) (33) \\
\hline IMGN388 & $\begin{array}{l}\alpha v \\
\text { integrin }\end{array}$ & DM4 & $\begin{array}{l}\text { GI tract (epithelial cells), bile duct, } \\
\text { melanocytes, kidney } \\
\text { tubules/glomeruli }\end{array}$ & $\begin{array}{l}\text { Gl toxicity, headache, } \\
\text { confusion (34) }\end{array}$ \\
\hline IMGN901 & CD56 & DM1 & $\begin{array}{l}\text { Peripheral nerves, neurons } \\
\text { (cerebellum), lung (epithelial cells), } \\
\text { glandular cells in GI, thyroid, and } \\
\text { adrenal }\end{array}$ & Peripheral neuropathy (35) \\
\hline PSMA ADC & PSMA & MMAE & $\begin{array}{l}\text { Kidney (proximal tubules) and } \\
\text { prostate }\end{array}$ & $\begin{array}{l}\text { Neutropenia, peripheral } \\
\text { neuropathy (36) }\end{array}$ \\
\hline SAR3419 & CD19 & DM4 & B cells & Ocular toxicity (keratitis) (37) \\
\hline SGN-35 & CD30 & MMAE & Activated lymphocytes & $\begin{array}{l}\text { Neutropenia; } \\
\text { thrombocytopenia; elevated } \\
\text { liver enzymes (38) }\end{array}$ \\
\hline SGN-75 & CD70 & MMAF & B cells and some $T$ cells & Neutropenia (39) \\
\hline T-DM1 & HER2 & DM1 & $\begin{array}{l}\text { Cardiac myocytes, epithelial cells in } \\
\text { GI tract, lung, skin, and breast }\end{array}$ & $\begin{array}{l}\text { Thrombocytopenia, elevated } \\
\text { liver enzymes (45-47) }\end{array}$ \\
\hline
\end{tabular}

Dose-limiting toxicities coded blue indicate findings in organs that express target (i.e., on-target); those coded red indicate findings in organs/ cells that do not express target (i.e., off-target)

$B M$ bone marrow, $L F T$ liver function tests, GI gastrointestinal 
regenerative potential of target cell/organ, mechanism/potency of warhead, and accessibility of the ADC to the target cell—could play a role.

\section{Off-Target Toxicity}

Off-target toxicity refers to adverse findings in organs/cells that do not express target. These toxicities are generally related to the safety profile of the free warhead and are common across a wide range of target antigens (referenced in Tables III and IV). Off-target toxicity is a major cause of dose-limiting toxicity (DLT) for ADCs in clinical development $(43,44)$. The maximum tolerated dose (MTD) for most ADCs conjugated to microtubule inhibitors has ranged from 2 to $5 \mathrm{mg} / \mathrm{kg}$ irrespective of target expression (7). Therefore, there is a great desire to improve TI of next generation ADCs, which can only be accomplished through greater understanding of the underlying mechanisms of off-target toxicity. The two major mechanisms of off-target toxicity are thought to be related to (1) conjugate stability and (2) nonspecific uptake into normal cells.

\section{Stability}

Instability of the conjugate-i.e., inappropriate release of the warhead outside target cells-leads to off-target toxicity by increasing systemic exposure to free warhead. Two of the major factors contributing to ADC stability are (1) choice of linker and (2) conjugation chemistry used to attach the warhead to the antibody (45).

Linker instability has been cited as a primary cause of failure of first-generation ADCs-including Mylotarg $®$, the first marketed ADC (46). The ideal linker should be sufficiently stable to deliver the ADC to the tumor site, yet labile enough to rapidly release the warhead inside the cell. Current-generation linkers are classified as either cleavable or noncleavable (45). The majority of cleavable linkers are designed to be selectively cleaved by enzymes in a low-pH environment such as a lysosome (47). This approach has been clinically validated by Adcetris ${ }^{\circledR}$, which uses a cleavable dipeptide linker that undergoes cathepsin B cleavage within the lysosome (48). Noncleavable linkers must undergo catabolic breakdown within the cell to release warhead. While these linkers have generally shown improved safety profiles compared to cleavable linkers, the overall impact on TI is variable as noncleavable ADCs often have less activity in tumor types with heterogenous target expression that require bystander effect (49). Presently, the relationship between linker choice/tumor type/efficacy/safety is not well understood; the optimal linker-warhead combination must be empirically tested for each ADC.

Another factor impacting stability is the type of conjugation chemistry used to attach the warhead linker to the $\mathrm{mAb}$. The most common approach to conjugation has been to nonselectively attach drugs to cysteine or lysine residues in the antibody via a reactive maleimide group (50). This process, also known as stochastic conjugation, produces a heterogenous mixture of ADCs with drug-antibody ratios (DARs) ranging from 0 to 8 (51). Heterogeneous DAR is thought to limit the TI of stochastic ADCs. Experiments with purified DAR species have demonstrated that higher DAR ADCs are more toxic than lower DAR ADCs at equivalent doses of antibody (52). This effect is thought to be related to faster clearance of higher DAR species. Recent advances in conjugation chemistry have led to the production of ADCs with homogeneous drug loading and improved stability (53). These technologies use nonnatural amino acids, engineered cysteines, or transglutaminases to attach the warhead linker to specific sites on the antibody $(54,55)$. The first published data using site-specific conjugation-i.e., THIOMAB technology-to generate homogenous DAR2 conjugates demonstrated significant improvements in TI compared to stochastically conjugated DAR4 ADCs (56). These sitespecific ADCs were less toxic in rats and monkeys while maintaining equivalent activity in tumor xenograft models. The improvements in TI were associated with slower clearance of the ADC and decreased deconjugation secondary to maleimide exchange (57). The first known ADC using site-specific technology in the clinic is SGNCD33A, an anti-CD33 antibody conjugated to highly potent pyrrolobenzodiazepine dimers via engineered cysteines (58). However, until clinical safety data is available, it remains unknown whether the improved TI with site-specific technology will translate to the clinic.

Table IV. Common Dose-Limiting Toxicities of ADCs

\begin{tabular}{lcccc}
\hline & & & Warhead & \\
\cline { 2 - 5 } Dose-limiting toxicity & MMAF & MMAE & Calich & DM1 \\
\hline Neutropenia & $\sqrt{ }$ & $\sqrt{ }$ & $\sqrt{ }$ \\
Thrombocytopenia & $\sqrt{ }$ & $\sqrt{ }$ & $\sqrt{ }$ \\
Peripheral neuropathy & & $\sqrt{ }$ & $\sqrt{ }$ \\
Elevated liver enzymes & & $\sqrt{ }$ \\
Grade 3/4 skin rash & & & $\sqrt{ }$ \\
Ocular toxicity & & & $\sqrt{ }$ \\
Grade 3/4 GI toxicity & & & $\sqrt{ }$ \\
Generalized symptoms & & & $\sqrt{ }$ \\
$\quad$ Headache, confusion, fatigue & & & $\sqrt{ }$ \\
Lymphopenia & & & \\
\hline
\end{tabular}

Common dose-limiting toxicities identified from ADCs listed in Table III

$M M A F$ monomethyl auristatin phenylalanine, MMAE monomethyl auristatin E, Calich calicheamicin, DM1 maytansine DM1, DM4 maytansine DM4 


\section{Target-Independent Uptake into Normal Cells}

While target-independent uptake of ADCs into normal cells is thought to be a major component of off-target toxicity, this is the least understood mechanism of ADCmediated toxicity. Antibodies are known to be taken up by normal cells in an antigen-independent manner by various mechanisms-including $\mathrm{Fc}$ receptor-mediated uptake (e.g., mannose receptors, FcRn, and Fc $\gamma \mathrm{R}$ receptors), nonspecific endocytosis, and/or uptake of ADC catabolites $(40,59)$. Theoretically, nonspecific uptake could lead to intracellular trafficking of the ADC through endosomal or lysosomal pathways, followed by release of the cytotoxic warhead inside normal cells. However, there is very little data to assess the impact of antigen-independent uptake on the overall off-target toxicity profile of ADCs. The most extensive data set to support this hypothesis is derived from a research study conducted to investigate the mechanism of T-DM1-mediated thrombocytopenia (60). This study was initiated following observations of a high incidence of dose-limiting thrombocytopenia in T-DM1 patients despite lack of target expression on platelets or platelet precursors $(42,61)$. Preliminary in vitro studies with platelet-rich plasma showed that T-DM1 does not have a direct effect on platelets. However, follow-up studies with megakaryocyte cultures demonstrated surface binding and internalization of Alexa488-conjugated T-DM1 in platelet precursors. FcR $\gamma \mathrm{IIb}$ appears to play a role in nonspecific uptake as preincubation with an anti-CD32 mAb decreased internalization of Alexa488conjugated T-DM1 by approximately twofold. However, Fc $\gamma$ receptor blocking experiments did not completely prevent $\mathrm{T}$ DM1 uptake by megakaryocytes, demonstrating that there are other nonFc $\gamma \mathrm{R}$ mechanisms involved (60). From a safety assessment standpoint, more work is needed to understand normal cell biodistribution and how engineering changes could impact the off-target safety profile of ADCs.

\section{CURRENT REGULATORY EXPECTATIONS FOR ADCS}

There are no regulatory guidelines that specifically address nonclinical safety testing of ADCs. While a Q\&A subsection pertaining to ADCs is planned for ICHS9, current guidance is limited to small subsections within ICHS6(R1) and S9 (2). The major principles addressed in these documents are species selection, stability (in vitro and in vivo), and relative importance of testing the conjugated $v s$. unconjugated material (outlined in Table V).

\section{Species Selection}

The principles of species selection are outlined in ICHS6(R1) (3). In general, species selection for a conjugated antibody should use the same approach as an unconjugated antibody-i.e., toxicity studies should only be conducted in relevant species with antibody cross-reactivity. As with most therapeutic mAbs, the high-affinity interaction between the ADC and its target usually precludes rodent cross-reactivity. In the situation where the rodent is not a relevant species, safety evaluation can be limited to a single nonrodent species-typically nonhuman primates due to high homology with the human target. In the case where the ADC does bind target in both rodents and nonrodents, toxicology studies must be conducted in two species (note 2). One aspect of species selection that is not covered by current guidelines is the utility of safety data generated in a nonrelevant species. For unconjugated mAbs, studies conducted in nonrelevant species-i.e., no target cross-reactivity-are considered misleading and are discouraged (3). This is due to the fact that most toxicities associated with therapeutic mAbs are related to excessive modulation of the primary pharmacological target (62). Therefore, in the absence of target engagement, toxicity studies with biopharmaceuticals do not produce any meaningful findings. In this scenario, other approaches-such as the minimum anticipated biological effect level-must be used to set the start dose (63). However, for ADCs, this argument does not hold true; the presence of a highly potent cytotoxic warhead produces significant toxicities that are unrelated to target binding (Table III). As a result, a toxicology conducted in a non cross-reactive species could provide relevant information as to the anticipated off-target toxicities and potentially be used to inform the clinical trial design and start dose (64).

\section{Stability}

As discussed previously, linker stability is one of the most important factors contributing to ADC safety (45). This is reflected in ICHS6(R1) and S9 guidelines, which state that stability and/or metabolic stability of ADCs should be addressed in human as well as test species plasma $(2,3)$. While scientific best practices for evaluating ADC stability have yet to be established, in vitro stability is typically assessed by determining loss of drug-to-antibody ratio (DAR) in human and test species plasma at $37^{\circ} \mathrm{C}$ for 3 to 7 days duration (57). To date, specifications for ADC stability have not been established; therefore, from a regulatory perspective, the primary objective of these studies is to establish that the data obtained from repeat dose toxicology studies is representative of the potential human situation.

In addition to in vitro assays, ADC stability must be further evaluated in vivo by measuring plasma levels of ADCs and their unconjugated components in test species. While ICHS9 clearly indicates that toxicokinetic evaluation of ADCs should assess both the conjugated and the unconjugated compound(s), it does not specify which of the individual components (i.e., total mAb, warhead, and/or linker) must be measured (2). This suggests that regulators could potentially accept PK data from less than three analytes (e.g., ADC and warhead only) in the good laboratory practice (GLP) toxicology studies. The decision to assay for multiple unconjugated components is further complicated by the fact that it is very challenging from a resource perspective to develop and validate multiple bioanalytical assays early in nonclinical development. However, bioanalytical difficulties aside, in vivo stability is best evaluated by measuring at least total ADC, total mAb (conjugated and unconjugated), and warhead (cleavable ADC) \pm linker (noncleavable ADC) in toxicology studies. This enables calculation of the deconjugation rate by comparing the concentration of total $\mathrm{mAb}$ to ADC in plasma (59). In addition, assaying for free warhead \pm linker can further enhance understanding of 
Table V. Regulatory Guidance Pertaining to Safety Assessment of Conjugated Products

\begin{tabular}{lll}
\hline & \multicolumn{1}{c}{ ICHS6(R1) } & \multicolumn{1}{c}{ ICHS9 $^{b}$} \\
\hline Species selection & Test ADC in relevant species & Not addressed \\
ADC stability & Assess in animal and human plasma & Assess in animal and human plasma \\
ADC toxicity & Full safety evaluation & Full safety evaluation \\
Warhead toxicity & Treat as an NME & "Limited" evaluation \\
Toxicokinetics & Not addressed & Assess conjugated and unconjugated forms \\
\hline
\end{tabular}

$I C H$ International Conference on Harmonization, $A D C$ antibody drug conjugates

${ }^{a}$ Section 2.1 and note 2 of ICHS6(R1)

${ }^{b}$ Section 4.1 of ICHS9

${ }^{c}$ If novel, treat as new molecular entity $(N M E)$. If previously tested, separate evaluation not necessary

conjugate instability by determining relative exposure to conjugated vs. free warhead. Therefore, the decision as to which components to should be included in the toxicokinetic analysis requires a weighted evaluation of the information needed to understand the nonclinical safety profile of ADCs.

\section{Relative Importance of Testing Conjugated vs. Unconjugated Material}

The only other principle specifically pertaining to the nonclinical safety assessment of ADCs in current guidelines is the relative importance of testing the conjugate $v s$. the unconjugated components in GLP toxicology studies. ICHS6(R1) and ICHS9 are both clear that the ADC should be the primary focus of the nonclinical safety evaluation. In addition, both guidelines agree that when the cytotoxic agent has been previously tested and there is a sufficient body of scientific information available, separate evaluation of the unconjugated warhead is not warranted $(2,3)$. Where the two guidelines differ-is in regard to the safety evaluation of the unconjugated components (i.e., unconjugated $\mathrm{mAb}$ and warhead). ICHS6(R1) advises that nonclinical safety assessment of novel cytotoxic warheads should follow the approach used for novel molecular entities (NME), as per ICHS9 recommendations. In contrast, ICHS9 states that toxicity testing of unconjugated components can have a more "limited" evaluation. This difference is significant. As it reads, following ICHS6(R1) would entail a full nonclinical toxicology package for a novel warhead that includes GLP toxicology studies in two species, safety pharmacology, genotoxicity assessment, and development and reproductive toxicology studies. From a regulatory perspective, it would seem likely that the recommendations in ICHS9 should outweigh those in ICHS6(R1) if the ADC is intended for use in patients with advanced cancer. This assumption is supported by marketing applications for Kadcyla ${ }^{\circledR}$ and Adcetris $\AA$, which did not contain a full nonclinical toxicology dataset for either of their warheads $(65,66)$. Following ICHS9 enables a flexible, science-based approach to safety testing of novel warheads that considers the unique properties of ADCs in the design of the nonclinical toxicology program.

The minimal data package of nonclinical safety studies needed to support an investigational new drug (IND) application for an ADC is outlined in Table VI.

\section{Safety Assessment of the Conjugate}

From a regulatory perspective, a GLP toxicology study with the ADC in a relevant species yields the primary data set used to inform the clinical start dose and identify potential human risks (2). As outlined in Table I of ICH S9, the duration of this study should be based on the intended dosing clinical schedule as outlined in (2). In general, a single-dose toxicity study with a 21-day observation period should be sufficient to support an every 3-week (Q3W) clinical dosing schedule unless there is a cause for concern (e.g., cumulative toxicity after repeated dosing). Safety pharmacology of the conjugate should also be assessed by incorporating safety pharmacology endpoints into the pivotal GLP study. Regulatory guidelines are also clear that a thorough assessment of ADC stability should be completed prior to IND submission (2).

\section{Safety Assessment of Novel Warheads}

The main purpose of characterizing the toxicity profile of the warhead is to evaluate the worst case scenario in the event that the molecule is fully unstable. Under these circumstances, a single-dose toxicology study in a rodent is expected to characterize the safety profile of the unconjugated warhead. However, in reality, it is more likely that the warhead will undergo slow release from the conjugate by target-mediated clearance and/or instability of the linker with altered biodistribution than undergo immediate release (5). While it would be ideal to conduct a toxicology study that mimics free warhead exposure following administration of the ADC, this would be near impossible to achieve from a practical standpoint. Therefore, the most relevant safety data is derived from toxicology studies with the conjugate. In addition, toxicology studies to evaluate the warhead alone can be limited to a single rodent species (64), as potent cytotoxics have such strong primary pharmacological activity that there are usually minimal species differences in the toxicity profile of these agents $(67,68)$. To complete the safety evaluation, the nonclinical package must also include a genotoxicity assessment of the novel warhead at the time of BLA submission.

Consideration must also be given to the need to conduct absorption, distribution, metabolism, and elimination (ADME) studies on novel cytotoxic warheads. While these studies are not required at the time of IND submission as per ICHS9, it is often advantageous to conduct a small screening study to evaluate efflux substrate potential and metabolic stability of novel warheads. These types of studies can minimize the risk of 
metabolic liabilities that could impact clinical development further down the line. As such, the decision to conduct ADME studies for novel warheads prior to first-in-human trials should be considered on a case-by-case basis.

\section{Safety Assessment of the Monoclonal Antibody}

Nonclinical safety evaluation of the unconjugated antibody is generally unwarranted. In theory, these studies could help evaluate the role of target biology in the safety profile of the conjugate. However, in practice, these studies are unlikely to yield decision-making data. As discussed earlier, mAbs used in ADCs are generally not selected for inherent effector function and are rather selected for their ability to undergo rapid internalization (18). In addition, ADCs are generally dosed on an intermittent schedule to allow for recovery from cytotoxic effects (19). In this scenario-i.e., a rapidly internalized $\mathrm{mAb}$ dosed on a Q3W schedule-a toxicology study with the unconjugated $\mathrm{mAb}$ is unlikely to produce significant toxicity. Therefore, given that most mAbs are only crossreactive in nonhuman primates (NHP), toxicology studies with the unconjugated antibody should generally be avoided unless there is a specific concern.

The studies outlined in Table VI will generate the safety data required to inform patients and clinicians about potential human safety risks of novel ADCs; however, they are not designed to address mechanistic questions related to ADC toxicity-e.g., "what impact does conjugation site, DAR, warhead mechanism of action, and/or target expression have on safety?" These types of questions are best addressed by investigative studies that can be applied across a company's ADC development platform and used to develop nextgeneration ADCs with improved TI.

\section{SAFETY STRATEGIES TO ADVANCE ADC DEVE LOPMENT}

\section{Early Assessment of On-Target Toxicity}

The major obstacle to early assessment of on-target toxicity is lack of antibody cross-reactivity to the target in rodents. As described above, NHP are usually the sole relevant species for mAbs. However, due to ethical and financial considerations, NHP toxicology studies are typically conducted later in the discovery process, i.e., after lead candidate selection. This leads to a great deal of uncertainty as the molecule progresses through the pipeline. Current mitigation strategies to de-risk on-target toxicity early in the discovery process are highly reliant on immunohistochemistry (IHC). This often produces a "bottleneck" as IHC protocol optimization is time-consuming and slides must be read by a trained pathologist. In addition, as described above, it is difficult to predict on-target toxicities based on target expression alone (Table III). Other key factors such as-vital nature of organ/cell type, regenerative potential of cell, anatomical location (membrane vs. cytoplasm, apical vs. basal), mechanism of action of warhead, and differential expression of target in tumor vs. normal cells-should also be considered. For example, safety assessment of on-target toxicity should always take into account the warhead's mechanism of action. Microtubule inhibitors-e.g., DM1, DM4, MMAE, and MMAF_-block mitosis of rapidly dividing cells by reversibly binding to microtubules (69). The reversible nature of this binding generally minimizes cytotoxicity to slowly dividing cells. Therefore, the proliferative index of the cell should be considered when evaluating the potential safety risks of a novel target-i.e., if the normal cell is a slow proliferator, it is less of a concern for on-target toxicity. This assumption is supported by existing nonclinical and clinical data demonstrating that the safety profile of ADCs is largely driven by the mechanism of action of the warhead (Table IV) $(40,70)$. It is unclear whether this relationship will hold true for novel warheads. There is a push within the field to use more potent warheads-e.g., DNA inhibitors-that target cancer stem cells. These agents, which target both slowly and rapidly dividing cells (71), have greater potential for unpredictable on-target toxicity. For example, use of a DNAdamaging warhead significant increased on-target toxicity in LGR5-expressing intestinal stem cells compared to a microtubule-inhibiting warhead (25). This potential for greater on-target toxicity necessitates use of more stringent criteria when selecting targets for highly potent ADCs as compared to first generation ADCs. Given the subjective nature of the current strategy, there is a clear need to develop better techniques to understand the impact of target expression in normal tissues. While rodent cross-reactive antibodies would enable early in vivo safety testing, generation of these molecules is often not feasible due to species differences in target homology (72). Other strategies would be to generate a

Table VI. Minimal Nonclinical Safety Package Required to Support IND and BLA Applications for ADCs

\begin{tabular}{llll}
\hline \multicolumn{1}{c}{ Study } & Molecule & Study duration & IND \\
\hline $\begin{array}{l}\text { GLP toxicology in relevant species } \\
\quad \text { (incl. safety pharmacology endpoints) }\end{array}$ & ADC & Single or repeat dose ${ }^{a}$ & $\sqrt{ }$ \\
GLP toxicology study in rat ${ }^{b}$ & & 3 months & $\sqrt{ }$ \\
In vitro plasma stability & Warhead & Single dose & $\sqrt{ }$ \\
Tissue cross-reactivity & ADC & N/A & $\sqrt{ }$ \\
Genotoxicity battery & ADC & & \\
Embryofetal development & Warhead & & $\sqrt{ }$ \\
\hline
\end{tabular}

$A D C$ antibody drug conjugates, $B L A$ biologics license application, $I N D$ investigational new drug, GLP good laboratory practice

${ }^{a}$ Refer to ICHS9 for additional details on study duration required to support IND applications

${ }^{b}$ If warhead has been previously tested and sufficient body of scientific information is available, a separate evaluation is not warranted

${ }^{c}$ If warhead is genotoxic and targets rapidly dividing cells, an embryofetal development toxicology study is not warranted (refer to ICHS9) 
rodent surrogate or to conduct a small scale NHP study to evaluate potential on-target toxicity early in development. However, these approaches involve ethical and practical considerations that must be weighed accordingly.

\section{Early Assessment of Novel ADC Technologies}

The biggest promise of ADCs is the potential to improve the TI of highly potent cytotoxic warheads using a targeted approach to increase tumor cell delivery and decrease systemic exposure. However, despite recent advances in the field, TI remains a key challenge to ADC development. There are many ongoing efforts to use protein and chemical engineering to further improve the stability and TI of ADCs. While these efforts are gaining momentum from an engineering perspective, the inability to predict the impact of these new technologies-i.e., novel conjugation chemistries, linkers, and warheads - on the safety and efficacy of ADCs continues to limit ADC development. Therefore, it is crucial to develop a strategic approach to candidate selection that enables rapid safety screening of new technologies early in the discovery process.

One such strategy is to use a rodent screening model to evaluate the impact of new technologies on the safety of novel ADC constructs. For the most part, new technologies-e.g., novel conjugation chemistries, linkers, and/or protein carriers-are being developed with the intention to increase TI by improving off-target toxicity. Therefore, safety assessment does not require use of a cross-reactive species as the findings are target-independent. Using a rodent screening model offers a cost-effective approach to rapid screening of the in vivo impact of novel technologies. An example of this approach is highlighted in a recent publication where a single-dose rat toxicology study was used to evaluate the impact of different conjugation sites on the safety and PK of a site-specific ADC (55). For the most part, these studies should be conducted in parallel to in vivo efficacy studies to assess the overall impact on TI. While this is just one possible strategy that can be considered, increased reliance on rodent models can speed up development by reducing test article demand/study costs.

\section{CONCLUSION}

Regulatory guidelines specify that a full nonclinical safety evaluation of the conjugate should be conducted to support first-in-human clinical trials with ADCs. The pivotal GLP toxicology studies should be conducted in a relevant species to evaluate potential on- and off-target safety risks. This study should include toxicokinetic analysis of both the conjugated and unconjugated components to assess the impact of stability on safety. There should also be an understanding of target expression, which can be derived from the GLP TCR study or available IHC data. Safety of evaluation of the warhead is more open to interpretation but should minimally include a single-dose rodent study. These data should be sufficient to set the clinical start dose and inform the clinical monitoring plan.

While these studies are required from the regulatory perspective, it is clear that the industry must continue to develop better safety strategies to drive the ADC field forward. Clinical development of ADCs continues to be limited by toxicity. Solving this problem can only be accomplished by filling in the gaps in our understanding of the mechanisms of ADC toxicity. One way to do so would be to employ a safety screening strategy to conduct early in vitro and in vivo mechanistic studies that are distinct from regulatory toxicology studies. While these studies are not required, they can (1) improve our understanding of the underlying mechanisms of toxicity, (2) accelerate ADC development, and (3) guide engineering efforts to improve TI of future ADCs. These efforts will in turn benefit both industry and patients as they will increase the chances of clinical success with next generation ADCs.

\section{ACKNOWLEDGMENTS}

The authors would like to thank Marlon Rebelatto, Xiang-Qing Yu, Andrew Pierce, Molly Reed, Shameen AfifRider, Joerg Bluemel, Christopher Frantz, Jay Harper, Song Ren, and Keith Steele.

\section{REFERENCES}

1. Mullard A. Maturing antibody-drug conjugate pipeline hits 30 . Nat Rev Drug Discov. 2013;12(5):329-32.

2. S9 nonclinical evaluation for anticancer pharmaceuticals. (2010).

3. Preclinical safety evaluation of biotechnology-derived pharmaceuticals S6(R1). (2011).

4. Roberts SA, Andrews PA, Blanset D, Flagella KM, Gorovits B, Lynch CM, et al. Considerations for the nonclinical safety evaluation of antibody drug conjugates for oncology. Regul Toxicol Pharmacol RTP. 2013;67(3):382-91.

5. Han TH, Zhao B. ADME considerations for the development of antibody-drug conjugates. Drug Metab Dispos Biol Fate Chemicals. 2014

6. Waldmann TA, Strober W. Metabolism of immunoglobulins. Prog Allergy. 1969;13:1-110.

7. Keizer RJ, Huitema AD, Schellens JH, Beijnen JH. Clinical pharmacokinetics of therapeutic monoclonal antibodies. Clin Pharmacokinet. 2010;49(8):493-507.

8. Deslandes A. Comparative clinical pharmacokinetics of antibody-drug conjugates in first-in-human phase 1 studies. mAbs. 2014;6(4):859-70.

9. Han TH, Zhao B. Absorption, distribution, metabolism, and excretion considerations for the development of antibody-drug conjugates. Drug Metab Dispos Biol Fate Chem. 2014;42(11):1914-20.

10. Erickson HK, Lewis Phillips GD, Leipold DD, Provenzano CA, Mai E, Johnson HA, et al. The effect of different linkers on target cell catabolism and pharmacokinetics/pharmacodynamics of trastuzumab maytansinoid conjugates. Mol Cancer Ther. 2012;11(5):1133-42.

11. Chari RV. Targeted cancer therapy: conferring specificity to cytotoxic drugs. Acc Chem Res. 2008;41(1):98-107.

12. Alley SC, Zhang X, Okeley NM, Anderson M, Law C-L, Senter $\mathrm{PD}$, et al. The pharmacologic basis for antibody-auristatin conjugate activity. J Pharmacol Exp Ther. 2009;330(3):932-8.

13. Shah DK, Betts AM. Towards a platform PBPK model to characterize the plasma and tissue disposition of monoclonal antibodies in preclinical species and human. J Pharmacokinet Pharmacodyn. 2012;39(1):67-86.

14. Xie H, Audette C, Hoffee M, Lambert JM, Blattler WA. Pharmacokinetics and biodistribution of the antitumor immunoconjugate, cantuzumab mertansine (huC242-DM1), and its two components in mice. J Pharmacol Exp Ther. 2004;308(3):1073-82.

15. Boswell CA, Mundo EE, Firestein R, Zhang C, Mao W, Gill H, et al. An integrated approach to identify normal tissue expression 
of targets for antibody-drug conjugates: case study of TENB2. Br J Pharmacol. 2013;168(2):445-57.

16. Henderson LA, Baynes JW, Thorpe SR. Identification of the sites of $\operatorname{IgG}$ catabolism in the rat. Arch Biochem Biophys. 1982;215(1):1-11.

17. Yip V, Palma E, Tesar DB, Mundo EE, Bumbaca D, Torres EK, et al., editors. Quantitative cumulative biodistribution of antibodies in mice: effect of modulating binding affinity to the neonatal Fc receptor. mAbs. Landes Bioscience; 2014.

18. Harper J, Mao S, Strout P, Kamal A. Selecting an optimal antibody for antibody-drug conjugate therapy: internalization and intracellular localization. Methods Mol Biol. 2013;1045:41-9.

19. Scott AM, Wolchok JD, Old LJ. Antibody therapy of cancer. Nat Rev Cancer. 2012;12(4):278-87.

20. Cheever MA, Allison JP, Ferris AS, Finn OJ, Hastings BM, Hecht TT, et al. The prioritization of cancer antigens: a national cancer institute pilot project for the acceleration of translational research. Clin Cancer Res Off J Am Assoc Cancer Res. 2009;15(17):5323-37.

21. Tijink BM, Buter J, de Bree R, Giaccone G, Lang MS, Staab A, et al. A phase I dose escalation study with anti-CD44v6 bivatuzumab mertansine in patients with incurable squamous cell carcinoma of the head and neck or esophagus. Clin Cancer Res Off J Am Assoc Cancer Res. 2006;12(20 Pt 1):6064-72.

22. Mackay C, Terpe H, Stauder R, Marston W, Stark H, Günthert $\mathrm{U}$. Expression and modulation of CD44 variant isoforms in humans. J Cell Biol. 1994;124(1):71-82.

23. Tolcher AW, Ochoa L, Hammond LA, Patnaik A, Edwards T, Takimoto $\mathrm{C}$, et al. Cantuzumab mertansine, a maytansinoid immunoconjugate directed to the $\mathrm{CanAg}$ antigen: a phase I, pharmacokinetic, and biologic correlative study. J Clin Oncol Off J Am Soc Clin Oncol. 2003;21(2):211-22.

24. Gudas GM, Torgov M, An Z, Jia XC, Morrison KJ, Morrison RK, et al. Use of AGS-16M8F as a novel antibody drug conjugate (ADC) for treating renal cancers. J Clin Oncol. 2010; 28. (suppl; abstr e15014)2010.

25. Klinguer-Hamour C, Strop P, Shah DK, Ducry L, Xu A, Beck A. World Antibody-Drug Conjugate Summit, October 15-16, 2013, San Francisco, CA. mAbs. 2014;6(1):18-29.

26. Coveler AL, Von Hoff D, Ko AH, Cherry Whiting N, Zhao B, Wolpin BM. A phase I study of ASG-5ME, a novel antibodydrug conjugate, in pancreatic ductal adenocarcinoma. J Clin Oncol. 2013; 31. (suppl 4; abstr 176).

27. Lapusan S, Vidriales MB, Thomas X, de Botton S, Vekhoff A, Tang R, et al. Phase I studies of AVE9633, an anti-CD33 antibody-maytansinoid conjugate, in adult patients with relapsed/refractory acute myeloid leukemia. Investig New Drugs. 2012;30(3):1121-31.

28. Kambham N, Kong C, Longacre TA, Natkunam Y. Utility of syndecan-1 (CD138) expression in the diagnosis of undifferentiated malignant neoplasms: a tissue microarray study of 1,754 cases. Appl Immunohistochem Mol Morphol AIMM Off Publ Soc Appl Immunohistochem. 2005;13(4):304-10.

29. Heffner LT, Jagannath S, Zimmerman TM, et al. BT062, an antibody-drug conjugate directed against CD138, shows clinical activity in patients with relapsed or relapsed/refractory multiple myeloma. Blood. 2011;118. Abstract 301.

30. Bendell J, Saleh M, Rose AA, Siegel PM, Hart L, Sirpal S, et al. Phase I/II study of the antibody-drug conjugate glembatumumab vedotin in patients with locally advanced or metastatic breast cancer. J Clin Oncol Off J Am Soc Clin Oncol. 2014;32(32):3619-25.

31. Advani A, Coiffier B, Czuczman MS, Dreyling M, Foran J, Gine $\mathrm{E}$, et al. Safety, pharmacokinetics, and preliminary clinical activity of inotuzumab ozogamicin, a novel immunoconjugate for the treatment of B-cell non-Hodgkin's lymphoma: results of a phase I study. J Clin Oncol Off J Am Soc Clin Oncol. 2010;28(12):2085-93.

32. Advani R, Lebovic D, Brunvand M, et al. A phase I study of DCDT2980S, an antibody-drug conjugate (ADC) targeting CD22, in relapsed or refractory B-cell non-Hodgkin's lymphoma. Blood. 2012;120(21):624. Abstract 59.

33. Qin A, Watermill J, Mastico RA, Lutz RJ. The pharmacokinetics and pharmacodynamics of IMGN242 (huC242-DM4) in patients with CanAg-expressing solid tumors. J Clin Oncol Off J Am Soc Clin Oncol. 2008;26(15S (May 20 Supplement)):3066.
34. Thompson DS, Patnaik A, Bendell JC, et al. A phase 1 doseescalation of IMGN388 in patients with solid tumors. J Clin Oncol Off J Am Soc Clin Oncol. 2010;28. (No 15_suppl (May 20 Supplement): 3058).

35. Woll P, Fossella F, O'Brien M, Clinch Y, Donaldson K, O'Keeffe J, et al. 510 POSTER phase I study of IMGN901 (BB-10901) in patients with CD56-positive solid tumours. EJC Suppl. 2008;6(12):162.

36. Petrylak DP, Smith D, Appleman LJ, et al. A phase II trial of prostate-specific membrane antigen antibody drug conjugate (PSMA ADC) in taxane-refractory metastatic castrationresistant prostate cancer (mCRPC). J Clin Oncol Off J Am Soc Clin Oncol. 2014;32. (suppl 4; abstr 83).

37. Ribrag V, Dupuis J, Tilly H, Morschhauser F, Laine F, Houot R, et al. A dose-escalation study of SAR3419, an anti-CD19 antibody maytansinoid conjugate, administered by intravenous infusion once weekly in patients with relapsed/refractory B-cell non-Hodgkin lymphoma. Clin Cancer Res Off J Am Assoc Cancer Res. 2014;20(1):213-20.

38. Pro B, Advani R, Brice P, Bartlett NL, Rosenblatt JD, Illidge T, et al. Brentuximab vedotin (SGN-35) in patients with relapsed or refractory systemic anaplastic large-cell lymphoma: results of a phase II study. J Clin Oncol Off J Am Soc Clin Oncol. 2012;30(18):2190-6.

39. Tannir NM, Forero-Torres A, Ramchandren R, Pal SK, Ansell SM, Infante JR, et al. Phase I dose-escalation study of SGN-75 in patients with CD70-positive relapsed/refractory non-Hodgkin lymphoma or metastatic renal cell carcinoma. Investig New Drugs. 2014;32(6):1246-57.

40. Poon KA, Flagella K, Beyer J, Tibbitts J, Kaur S, Saad O, et al. Preclinical safety profile of trastuzumab emtansine (T-DM1): mechanism of action of its cytotoxic component retained with improved tolerability. Toxicol Appl Pharmacol. 2013;273(2):298-313.

41. Barginear MF, John V, Budman DR. Trastuzumab-DM1: a clinical update of the novel antibody-drug conjugate for HER2overexpressing breast cancer. Mol Med. 2012;18(1):1473-9.

42. Krop IE, LoRusso P, Miller KD, Modi S, Yardley D, Rodriguez G, et al. A phase II study of trastuzumab emtansine in patients with human epidermal growth factor receptor 2-positive metastatic breast cancer who were previously treated with trastuzumab, lapatinib, an anthracycline, a taxane, and capecitabine. J Clin Oncol Off J Am Soc Clin Oncol. 2012;30(26):3234-41.

43. Baumann A, Flagella K, Forster R, de Haan L, Kronenberg S, Locher M, et al. New challenges and opportunities in nonclinical safety testing of biologics. Regul Toxicol Pharmacol RTP. 2014;69(2):226-33.

44. Sassoon I, Blanc V. Antibody-drug conjugate (ADC) clinical pipeline: a review. Methods Mol Biol. 2013;1045:1-27.

45. Nolting B. Linker technologies for antibody-drug conjugates. Methods Mol Biol. 2013;1045:71-100.

46. Doronina SO, Toki BE, Torgov MY, Mendelsohn BA, Cerveny CG, Chace DF, et al. Development of potent monoclonal antibody auristatin conjugates for cancer therapy. Nat Biotechnol. 2003;21(7):778-84.

47. Perez HL, Cardarelli PM, Deshpande S, Gangwar S, Schroeder GM, Vite GD, et al. Antibody-drug conjugates: current status and future directions. Drug Discov Today. 2014;19(7):869-81.

48. Younes A, Bartlett NL, Leonard JP, Kennedy DA, Lynch CM, Sievers EL, et al. Brentuximab vedotin (SGN-35) for relapsed CD30-positive lymphomas. N Engl J Med. 2010;363(19):1812-21.

49. Polson AG, Calemine-Fenaux J, Chan P, Chang W, Christensen E, Clark S, et al. Antibody-drug conjugates for the treatment of non-Hodgkin's lymphoma: target and linker-drug selection. Cancer Res. 2009;69(6):2358-64.

50. Senter PD. Potent antibody drug conjugates for cancer therapy. Curr Opin Chem Biol. 2009;13(3):235-44.

51. Panowksi S, Bhakta S, Raab H, Polakis P, Junutula JR. Sitespecific antibody drug conjugates for cancer therapy. mAbs. 2014;6(1):34-45.

52. Hamblett KJ, Senter PD, Chace DF, Sun MM, Lenox J, Cerveny $\mathrm{CG}$, et al. Effects of drug loading on the antitumor activity of a monoclonal antibody drug conjugate. Clin Cancer Res Off J Am Assoc Cancer Res. 2004;10(20):7063-70.

53. Behrens CR, Liu B. Methods for site-specific drug conjugation to antibodies. mAbs. 2014;6(1):46-53. 
54. Jackson D, Atkinson J, Guevara CI, Zhang C, Kery V, Moon SJ, et al. In vitro and in vivo evaluation of cysteine and site specific conjugated herceptin antibody-drug conjugates. PLoS One. 2014;9(1), e83865.

55. Strop P, Liu SH, Dorywalska M, Delaria K, Dushin RG, Tran TT, et al. Location matters: site of conjugation modulates stability and pharmacokinetics of antibody drug conjugates. Chem Biol. 2013;20(2):161-7.

56. Junutula JR, Raab H, Clark S, Bhakta S, Leipold DD, Weir S, et al. Site-specific conjugation of a cytotoxic drug to an antibody improves the therapeutic index. Nat Biotechnol. 2008;26(8):925-32.

57. Shen BQ, Xu K, Liu L, Raab H, Bhakta S, Kenrick M, et al. Conjugation site modulates the in vivo stability and therapeutic activity of antibody-drug conjugates. Nat Biotechnol. 2012;30(2):184-9.

58. Kung Sutherland MS, Walter RB, Jeffrey SC, Burke PJ, Yu C, Kostner H, et al. SGN-CD33A: a novel CD33-targeting antibodydrug conjugate using a pyrrolobenzodiazepine dimer is active in models of drug-resistant AML. Blood. 2013;122(8):1455-63.

59. Gorovits B, Krinos-Fiorotti C. Proposed mechanism of off-target toxicity for antibody-drug conjugates driven by mannose receptor uptake. Cancer Immunol Immunother CII. 2013;62(2):217-23.

60. Mahapatra K, Darbonne W, Bumbaca D, Shen B, Du X, Tibbitts J, et al. Abstract A135: T-DM1-induced thrombocytopenia results from impaired platelet production in a HER 2 -independent manner. Mol Cancer Ther. 2011;10(Supplement 1):A135.

61. Press MF, Cordon-Cardo C, Slamon DJ. Expression of the HER$2 /$ neu proto-oncogene in normal human adult and fetal tissues. Oncogene. 1990;5(7):953-62.
62. Kramer JA, Sagartz JE, Morris DL. The application of discovery toxicology and pathology towards the design of safer pharmaceutical lead candidates. Nat Rev Drug Discov. 2007;6(8):636-49.

63. Agency EM. Guideline on requirements for first in man clinical trials for potential high-risk mediciinal products 2007. Available from: http://www.ema.europa.eu/docs/en_GB/document_library/ Scientific_guideline/2009/09/WC500002989.pdf.

64. Saber H, Leighton JK. An FDA oncology analysis of antibodydrug conjugates. Regul Toxicol Pharmacol. 2015;71(3):444-52.

65. Pharmacology/toxicology BLA review: adcetris 2011. Available from: http://www.accessdata.fda.gov/drugsatfda_docs/nda/2011/ 125388Orig1s000PharmR.pdf.

66. Pharmacology and toxicology BLA review: Kadcyla 2013. Available from: http://www.accessdata.fda.gov/drugsatfda_docs/ nda/2013/125427Orig1s000PharmR.pdf.

67. Greaves P, Williams A, Eve M. First dose of potential new medicines to humans: how animals help. Nat Rev Drug Discov. 2004;3(3):226-36.

68. Sapra P, Shor B. Monoclonal antibody-based therapies in cancer: advances and challenges. Pharmacol Ther. 2013;138(3):452-69.

69. Lodish H, Berk A, Zipursky SL, et al. Microtubule dynamics and associated proteins. Molecular cell biology. 4th ed. New York: W. H. Freeman; 2000.

70. Trail PA. Antibody drug conjugates as cancer therapeutics. Antibodies. 2013;2(1):113-29.

71. Ritter A. Antibody-drug conjugates. Pharm Technol. 2012;36(1):42-7.

72. Chapman K, Pullen N, Graham M, Ragan I. Preclinical safety testing of monoclonal antibodies: the significance of species relevance. Nat Rev Drug Discov. 2007;6(2):120-6. 